PROCEEDINGS OF THE AMERICAN MATHEMATICAL SOCIETY

Volume 124, Number 2, February 1996

\title{
SOBOLEV IMBEDDING THEOREMS IN BORDERLINE CASES
}

\author{
NICOLA FUSCO, PIERRE LOUIS LIONS, AND CARLO SBORDONE \\ (Communicated by Barbara Lee Keyfitz)
}

\begin{abstract}
An imbedding theorem is given for functions whose gradient belongs to a class slightly larger than $L^{n}(\Omega), \Omega \subset \mathbb{R}^{n}$.
\end{abstract}

\section{INTRODUCTION}

We wish here to come back to the well-known imbedding theorem for $W^{1, n}(\Omega)$ functions due to N. Trudinger [T] and J. Moser [Mo].

One way to shed some light on the phenomena involved consists in extending this result to other spaces closely related to $W^{1, n}$, as in [ALT].

We consider here spaces of functions that are larger than $W^{1, n}$, but are contained in $\bigcap_{1<p<n} W^{1, p}$. More precisely, we consider functions $u$ whose gradient $D u$ satisfies for some $\sigma>0$

$$
\int_{\Omega}|D u|^{n} \log ^{-\sigma}(e+|D u|) d x<\infty
$$

a class of functions motivated by recent work on the regularity properties of Jacobians (see [BFS], [CLMS], [Mu], [IS], [Gr]).

In this paper we prove, in particular, that if $u \in W_{0}^{1,1}$ and (1) holds, then

$$
\int_{\Omega} \exp \left(\frac{|u(x)|^{\alpha}}{\lambda}\right) d x<\infty
$$

for any $\lambda>0$, where $\alpha=\frac{n}{n-1+\sigma}$.

This result relies on the simple observation that condition (2) is equivalent to saying that

$$
\lim _{p \rightarrow \infty} \frac{1}{p}\left({ }_{\Omega}|u|^{\alpha p} d x\right)^{1 / p}=0
$$

(where ${ }_{\Omega}$ stands for $\frac{1}{|\Omega|} \int_{\Omega}$ ).

More generally, our results apply to functions such that

$$
\sup _{\varepsilon>0} \varepsilon_{\Omega}^{\sigma}|D u|^{n-\varepsilon} d x<\infty
$$

(see Theorem 2).

Received by the editors July 7, 1993 and, in revised form, September 12, 1994.

1991 Mathematics Subject Classification. Primary 46E35.

(C)1996 American Mathematical Society 


\section{SOME PRELIMINARY RESULTS}

Let $\Omega$ be an open set in $\mathbb{R}^{n}$, with finite measure $|\Omega|$. We denote by $\operatorname{EXP}=$ $\operatorname{EXP}(\Omega)$ the set of functions $g: \Omega \rightarrow \mathbb{R}$ such that there exists $\lambda>0$ for which

$$
\exp _{\Omega}\left(\frac{|g|}{\lambda}\right) d x<\infty .
$$

One way to test if a function $f$ belongs to EXP is given by the following proposition, whose proof is an easy exercise (see [G, Chapter VI, Example 17]).

Proposition 1. Let $g: \Omega \rightarrow \mathbb{R}$ be a measurable function. Set

$$
E(g)=e \limsup _{p \rightarrow \infty} \frac{1}{p}\left({ }_{\Omega}|g|^{p} d x\right)^{1 / p}
$$

Then

$$
E(g)=\inf \left\{\lambda>0: \quad \exp \left(\frac{|g|}{\lambda}\right) d x<\infty\right\} .
$$

Remark. This proposition says that $g \in$ EXP if and only if $E(g)<\infty$. In particular, if $f \in L^{\infty}(\Omega)$ we have $E(f)=0$ and $E(g-f)=E(g)$ for any $g \in$ EXP.

We recall that EXP is a Banach space under the norm (see $[\mathrm{RR}]$ )

$$
\|g\|_{\mathrm{EXP}}=\inf \left\{\lambda>0: \quad \exp \left(\frac{|g|}{\lambda}\right) d x \leq 2\right\} .
$$

We also remark that $L^{\infty}$ is not a dense subspace of EXP. Indeed one can easily prove the following

Proposition 2. $E(g)=0$ if and only if there exists a sequence $f_{h} \in L^{\infty}$ such that $\left\|f_{h}-g\right\|_{\mathrm{EXP}} \rightarrow 0$.

Proof. If $f_{h}$ verifies $\left\|f_{h}-g\right\|_{\mathrm{EXP}} \rightarrow 0$, then by the previous remark:

$$
E(g)=E\left(f_{h}-g\right) \leq\left\|f_{h}-g\right\|_{\mathrm{EXP}} .
$$

Conversely, assume that $E(g)=0$, and let $\varepsilon>0$. By definition, we have

$$
\exp _{\Omega}\left(\frac{|g|}{\varepsilon}\right) d x<\infty
$$

then there exists $h_{\varepsilon}$ such that, for $h>h_{\varepsilon}$,

If we set

$$
\frac{1}{|\Omega|} \int_{\{|g|>h\}} \exp \left(\frac{|g|}{\varepsilon}\right) d x \leq 1
$$

$$
f_{h}(x)= \begin{cases}g(x) & \text { if }|g(x)| \leq h \\ 0 & \text { otherwise }\end{cases}
$$

then

$$
\exp _{\Omega}\left(\frac{\left|f_{h}-g\right|}{\varepsilon}\right) d x=\frac{1}{|\Omega|} \int_{\{|g|>h\}} \exp \left(\frac{|g|}{\varepsilon}\right) d x+\frac{|\{|g| \leq h\}|}{|\Omega|} \leq 2 .
$$

Therefore we have

$$
\left\|f_{h}-g\right\|_{\mathrm{EXP}} \leq \varepsilon \quad \text { for } h>h_{\varepsilon}
$$




\section{The MAIN RESUlts}

The first Sobolev type result we want to deduce is the following

Theorem 1. If $u \in W_{0}^{1,1}(\Omega)$ satisfies for some $\sigma>0$ the condition

$$
\lim _{\varepsilon \rightarrow 0^{+}} \varepsilon_{\Omega}^{\sigma}|D u|^{n-\varepsilon} d x=0
$$

then, for any $c>0$, we have

$$
\operatorname{sexp}_{\Omega}\left(\frac{|u|^{\alpha}}{c}\right) d x<\infty
$$

with $\alpha=\frac{n}{n-1+\sigma}$, i.e. $E\left(|u|^{\alpha}\right)=0$.

Let us recall that the Riesz potential If of a function $f \in L^{1}(\Omega)$ is defined as

$$
\operatorname{If}(x)=\int_{\Omega} f(y)|x-y|^{1-n} d y .
$$

We will use the following theorem ([GT, Theorem 7.34]).

Theorem. Let $1 \leq p, q \leq \infty$ and assume that $0 \leq \delta=\frac{1}{p}-\frac{1}{q}<\frac{1}{n}$. Then

$$
\|I f\|_{L^{q}(\Omega)} \leq\left(\frac{1-\delta}{\frac{1}{n}-\delta}\right)^{1-\delta} \omega_{n}^{1-1 / n}|\Omega|^{1 / n-\delta}\|f\|_{L^{p}(\Omega)}
$$

where $\omega_{n}$ is the measure of the unit ball in $\mathbb{R}^{n}$.

We can now pass to the

Proof of Theorem 1. Since

$$
|u(x)| \leq \frac{1}{n \omega_{n}} I(|D u|)(x),
$$

using Proposition 1, it will be enough to prove that

$$
\lim _{\varepsilon \rightarrow 0^{+}} \varepsilon^{1 / \alpha}\left({ }_{\Omega}[I(|D u|)]^{1 / \varepsilon} d x\right)^{\varepsilon}=0 .
$$

In (5) let us take $q=\frac{1}{\varepsilon}, p=n-\varepsilon$, and note that, if $0<\varepsilon \leq \frac{1}{n}$, then certainly $\delta<\frac{1}{n}$; hence we have

$$
\varepsilon^{1 / \alpha}\left({ }_{\Omega}[I(|D u|)]^{1 / \varepsilon} d x\right)^{\varepsilon} \leq \varepsilon^{1 / \alpha}\left(\frac{1-\delta}{\frac{1}{n}-\delta}\right)^{1-\delta} \omega_{n}^{1-1 / n}|\Omega|^{1 / n-\delta-\varepsilon}\|D u\|_{L^{n-\varepsilon}}(\Omega) .
$$

It is easy to check that, if $0<\varepsilon \leq \frac{1}{n}$, then

$$
\left(\frac{1-\delta}{\frac{1}{n}-\delta}\right)^{1-\delta} \leq c(n) \varepsilon^{-(n-1) / n}
$$

so we have

$$
\begin{aligned}
& \varepsilon^{1 / \alpha}\left({ }_{\Omega}[I(|D u|)]^{1 / \varepsilon} d x\right)^{\varepsilon} \\
& \quad \leq c(n,|\Omega|) \varepsilon^{1 / \alpha-(n-1) / n} \varepsilon^{-\sigma /(n-\varepsilon)}\left(\varepsilon_{\Omega}^{\sigma}|D u|^{n-\varepsilon} d x\right)^{1 /(n-\varepsilon)} \\
& \quad \leq c(n,|\Omega|) \varepsilon^{1 / \alpha-(n-1) / n-\sigma / n}\left(\varepsilon_{\Omega}^{\sigma}|D u|^{n-\varepsilon} d x\right)^{1 /(n-\varepsilon)}
\end{aligned}
$$


which proves (6), since $\frac{1}{\alpha}-\frac{n-1}{n}-\frac{\sigma}{n}=0$.

Remark 1. If $g \in L_{\text {loc }}^{1}(\Omega)$ satisfies

$$
\Omega_{\Omega}^{|g|^{n} \log ^{-\sigma}(e+g) d x<+\infty}
$$

then (see [BFS, Lemma 3])

$$
\lim _{\varepsilon \rightarrow 0^{+}} \varepsilon_{\Omega}^{\sigma}|g|^{n-\varepsilon} d x=0 .
$$

From this it follows that if $u \in W_{0}^{1,1}(\Omega)$ and $|D u|^{n} \log ^{-\sigma}(e+|D u|) \in L^{1}(\Omega)$, then

$$
\Omega_{\Omega} \exp \left(\frac{|u|^{\alpha}}{c}\right)<\infty
$$

for any $c>0$, where $\alpha$ is given by Theorem 1 . Note that, in any dimension, if $\sigma=1$, then $\alpha=1$.

Remark 2. Theorem 1 is optimal in the sense that the exponent $\alpha$ cannot be improved. In fact, if

$$
u(x)=\frac{\log |x|}{|\log | \log |x|||^{\vartheta}}
$$

for $|x|$ small, with $\vartheta>1 / n$, then $|D u|^{n} \log ^{-1}(e+|D u|)$ is in $L^{1}$, while, for any $c>0, \delta>0$

$$
\int \exp \left(\frac{|u|^{1+\delta}}{c}\right)=\infty
$$

We conclude by proving an imbedding theorem in terms of the quantity on the left-hand side of (3), which can be regarded as an extension of Trudinger's imbedding theorem.

Theorem 2. Let $u \in W_{0}^{1,1}(\Omega)$ satisfy for some $\sigma \geq 0$

$$
M=\sup _{0<\varepsilon \leq 1}\left(\varepsilon^{\sigma}|D u|^{n-\varepsilon} d x\right)^{1 /(n-\varepsilon)}<\infty .
$$

Then, if $\alpha=\frac{n}{n-1+\sigma}$, there exist $c_{1}=c_{1}(n, \sigma), c_{2}=c_{2}(n, \sigma)$ such that

$$
\exp _{\Omega}\left(\frac{|u|}{c_{1} M|\Omega|^{1 / n}}\right)^{\alpha} d x \leq c_{2} .
$$

Proof. Arguing as in the proof of Theorem 1, we have for any $0<\varepsilon \leq \frac{1}{n}$

$$
\begin{aligned}
\varepsilon^{1 / \alpha}\left({ }_{\Omega}|u(x)|^{1 / \varepsilon}\right)^{\varepsilon} & \leq \frac{\varepsilon^{1 / \alpha}}{n \omega_{n}}\left({ }_{\Omega}[I(|D u|)]^{1 / \varepsilon} d x\right)^{\varepsilon} \\
& \leq c(n) \varepsilon^{1 / \alpha-(n-1) / n}|\Omega|^{1 / n}\left(\varepsilon_{\Omega}^{\sigma}|D u|^{n-\varepsilon} d x\right)^{1 /(n-\varepsilon)} \\
& \leq c(n) \varepsilon^{1 / \alpha+1 / n-1-\sigma / n}|\Omega|^{1 / n} M \\
& =c(n)|\Omega|^{1 / n} M .
\end{aligned}
$$


From this inequality, taking $\alpha \varepsilon=\frac{1}{p}$, we get

$$
\sup _{p \geq \frac{n}{\alpha}} \frac{1}{p}\left({ }_{\Omega}\left(|u|^{\alpha}\right)^{p} d x\right)^{1 / p} \leq\left[c(n)|\Omega|^{1 / n} M\right]^{\alpha} .
$$

The result now follows, noting that if

$$
\lambda=\sup _{p \geq p_{0}} \frac{1}{p}\left({ }_{\Omega}|u|^{p} d x\right)^{1 / p},
$$

where $p_{0} \geq 1$, then

$$
\exp _{\Omega}\left(\frac{|u|}{2 e \lambda}\right) d x \leq c\left(p_{0}\right) .
$$

Remark 3. If $|D u| \in L^{n, \infty}=$ weak- $L^{n}$, then (7) holds with $\sigma=1$ (see [IS]) and then (8) holds with $\alpha=1$. For this and similar results, see [ALT]. Note that Theorem 2 holds true also in the case $\sigma=0$. In this case the above result reduces to Trudinger's theorem (see $[\mathrm{T}]$ ).

\section{REFERENCES}

[ALT] A. Alvino, P. L. Lions, and G. Trombetti, On optimization problems with prescribed rearrangements, Nonlinear Anal. TMA 13 (1989), 185-220. MR 90c:90236

[BFS] H. Brèzis, N. Fusco, and C. Sbordone, Integrability for the Jacobian of orientation preserving mappings, J. Funct. Anal. 115 (1993), 425-431. MR 94j:26024

[CLMS] R. Coifman, P. L. Lions, Y. Meyer, and S. Semmes, Compensated compactness and Hardy spaces, J. Math. Pures Appl. 72 (1993), 247-286. MR 95d:46033

[G] J. B. Garnett, Bounded analytic functions, Academic Press, New York, 1981. MR 83g:30037

[GT] D. Gilbarg and N. S. Trudinger, Elliptic partial differential equations of second order, Springer, Berlin and New York, 1977. MR 57:13109

[Gr] L. Greco, A remark on the equality $\operatorname{det} D f=\operatorname{Det} D f$, Differential and Integral Equations 6 (1993), 1089-1100. MR 95a:49104

[IS] T. Iwaniec and C. Sbordone, On the integrability of the Jacobian under minimal hypotheses, Arch. Rational Mech. Anal. 119 (1992), 129-143. MR 93i:49057

[Mo] J. Moser, A sharp form of an inequality by N. Trudinger, Indiana Univ. Math. J. 20 (1971), 1077-1092. MR 46:662

$[\mathrm{Mu}] \quad \mathrm{S}$. Müller, Higher integrability of determinants and weak convergence in $L^{1}$, J. Reine Angew. Math. 412 (1990), 20-34. MR 92b:49026

[RR] M. M. Rao and Z. D. Ren, Theory of Orlicz spaces, Marcel Dekker, New York, 1991. MR 92e: 46059

[T] N. S. Trudinger, On imbeddings into Orlicz spaces and some applications, J. Math. Mech. 17 (1967), 473-483. MR 35:7121

(N. Fusco and C. Sbordone) Dipartimento di Matematica e Applicazioni, Università di Napoli, via Cintia, 80126 Napoli, Italy

(P. L. Lions) Ceremade, Place du Marèchal de Lattre de Tassigny, 75775 Paris Cedex 16, France 